\title{
An Adaptive Information Quantity-Based Broadcast Protocol for Safety Services in VANET
}

\author{
Wenjie Wang, Tao Luo, and Ying Hu \\ Beijing Key Laboratory of Network System Architecture and Convergence, Beijing Laboratory of Advanced Information Networks, \\ Beijing University of Posts and Telecommunications, Beijing 100876, China
}

Correspondence should be addressed to Wenjie Wang; isa_guet@163.com

Received 17 June 2016; Revised 19 September 2016; Accepted 16 October 2016

Academic Editor: Seok-Chul Kwon

Copyright (C) 2016 Wenjie Wang et al. This is an open access article distributed under the Creative Commons Attribution License, which permits unrestricted use, distribution, and reproduction in any medium, provided the original work is properly cited.

\begin{abstract}
Vehicle-to-vehicle communication plays a significantly important role in implementing safe and efficient road traffic. When disseminating safety messages in the network, the information quantity on safety packets changes over time and space. However, most of existing protocols view each packet the same to disseminate, preventing vehicles from collecting more recent and precise safety information. Hence, an information quantity-based broadcast protocol is proposed in this paper to ensure the efficiency of safety messages dissemination. In particular, we propose the concept of emergency-degree to evaluate packets' information quantity. Then we present EDCast, an emergency-degree-based broadcast protocol. EDCast differentiates each packet's priority for accessing the channel based on its emergency-degree so as to provide vehicles with more safety information timely and accurately. In addition, an adaptive scheme is presented to ensure fast dissemination of messages in different network condition. We compare the performance of EDCast with those of three other representative protocols in a typical highway scenario. Simulation results indicate that EDCast achieves higher broadcast efficiency and less redundancy with less delivery delay. What we found demonstrates that it is feasible and necessary for incorporating information quantity of messages in designing an efficient safety message broadcast protocol.
\end{abstract}

\section{Introduction}

The Vehicular ad hoc Network (VANET) is a specific application for wireless communication technology implementing in Intelligent Transportation System (ITS) [1] to improve the safety and efficiency of road traffic. In the United States, a 75 $\mathrm{MHz}$ bandwidth at the $5.9 \mathrm{GHz}$ band [2] has been allocated to support the Dedicated Short-Range Communication (DSRC) [3] for supporting various applications in VANET. Moreover, the 802.11p standard defines specifications of the physical layer and the Medium Access Control (MAC) layer of Vehicleto-Vehicle (V2V) or Vehicle-to-Roadside (V2R) communications. Lots of safety applications are available for satisfying the requirement of ITS, such as accident warning, collision detecting, and intelligent driving [4]. These safety services guide drivers to take some action, so as to improve the safety and quality of road transportation. Since the communication range of vehicles is limited between $300 \mathrm{~m}$ and $400 \mathrm{~m}$, lifecritical safety messages need to be broadcasted by multihop to cover the Region of Interest (RoI), for example, $1000 \mathrm{~m}$.
Different from transmitting nonsafety messages in unicast or geomulticast, broadcasting safety messages requires stricter Quality of Service (QoS), for example, small latency and high reliability. Besides, because of lack of Request to Send/Clear to Send (RTS/CTS) scheme in the current scheme of IEEE802.11p, the failure of transmission often occurs because of weak links or frequent collisions with hidden terminals. What is more, there is no acknowledgment (ACK) scheme to detect the transmission failure, and no backoff mechanism is used to alleviate the newly coming collisions even in the sparse condition, which will significantly degrade the broadcast performance. Moreover, VANET is characterized by uneven vehicle distribution and highly dynamic topology. Therefore, how to broadcast the safety messages quickly and reliably is a challenging work, attracting increasing attention from industrial and academic communities.

To disseminate safety-related messages, rich broadcast protocols $[4,5]$ have been proposed, such as waiting-based 
protocols [6-10], probability-based protocols [11, 12], and contention-based protocols $[13,14]$. Their common ideas are farthest-first, allowing the farthest vehicles to rebroadcast packets first. Take waiting-based protocols as an instance, vehicles that locate farther away from themselves to sender are assigned higher priority to rebroadcast packets in terms of less waiting time, so as to achieve greater hop progress and less end-to-end delay.

However, most of existing broadcast protocols view all messages equally and overtake their different information quantity. For example, a newly produced emergency message contains more information quantity than an old message. However, they are assigned with the same size of Contention Window (CW) by most contention-based protocols. Another example is that waiting-based protocols give higher priority to the message from a farther location while vehicles actually prefer the message generated in a nearby station.

In this work, we define the concept of information quantity of messages in VANET and explore the benefits of integrating information quantity into the design of safety data broadcast protocol. Information quantity is an index of emergency level of messages, which indicates the safety status of road and traffic. The more emergent event a message represents, the more information quantity it contains. Since the messages with more information quantity are more useful to the vehicles driving towards where the messages take place, the higher priority should be assigned to them for channel access. Therefore, we propose the concept emergency-degree (ED) to vividly evaluate the information quantity of each message. Then an emergency-degree-based safety messages broadcast protocol, EDCast, is designed. Packets with higher ED values are assigned higher priorities in terms of a higher broadcast probability and a smaller size of CW. Besides, an adaptive scheme based on estimated vehicle density is adopted to optimize delay performance.

The main contributions of this paper are as follows:

(1) We propose the concept of emergency-degree (ED) to quantify the information quantity of different messages. ED incorporates three message attributes, which are temporal information quantity, spatial information quantity, and type information quantity. Spatial information quantity depends on cumulative number of hops (hop in italic means the number of hops in this paper) and the overall covering distances from generation to current time. To the best of our knowledge, this is the first attempt to mathematically evaluate the dynamic information quantity of messages in VANET.

(2) EDCast, an adaptive emergency-degree-based broadcast protocol, is proposed, which gives greater opportunities to more urgent messages to disseminate quickly. Compared to three other representative broadcast protocols in a typical highway scenario using NS2 simulator, EDCast performs better in terms of broadcast efficiency and delivery delay. It also reaches higher emergency packet delivery ratio while ensuring fast dissemination of emergency messages.
The rest of this paper is organized as follows. We review the related works in Section 2. We discuss our motivation and challenges in Section 3. We present metrics of information quantity in Section 4. We propose EDCast protocol in detail in Section 5 and discuss its performance in Section 6. Finally the conclusions are drawn in Section 7.

\section{Related Works}

In our previous work [15], we have summarized by simulation that receiver-based protocols outperform senderbased protocols due to high reliability, little overhead, and completely distributed manner. Mflood [16] is one of the most common receiver-based protocols. Although it provides fast and reliable dissemination, the broadcast storm [6] in dense network would significantly degrade broadcast performance. To mitigate broadcast storm, many valuable protocols have been proposed, including waiting-based, probability-based, and contention-based schemes.

The basic idea of waiting-based protocols is to differentiate candidates' waiting time, which is inversely proportional to the distance from receivers to last forwarder. Slotted1 [7] assigned vehicles in farthest segments waiting less time before rebroadcast. Similarly, a binary-partition-assisted broadcast (BPAB) [8] was proposed to improve the latency performance using a binary-partition scheme. To obtain hop progress and reduce redundancy, eSBR [9] were present to help receivers make forwarding decisions according to the one-hop covering range. In addition, a two-phase broadcast scheme OppCast was proposed [17]. One phase satisfies fast dissemination while the other phase ensures reliability. Recently, ROFF [10] was proposed to mitigate collision by considering the short difference of waiting time of adjacent vehicles.

Probability-based schemes allow parts of receivers to forward packets to reduce redundancy. In slotted-p and weighted-p [7], vehicles that are farther away from the previous forwarder have higher probabilities to rebroadcast packets, so that the hop progress would be maximized and the end-to-end delay would be minimized. The forwarding probability of packets in [11] was given based on the number of duplicates received by vehicles, while in [12] the probability relies on the location and the density of network. In this way, the collision is reduced significantly.

Different from waiting-based schemes, contention-based schemes differentiate packets' priorities by adjusting the size of CW rather than using a timer. In [13], a priority setting scheme and a collision avoidance mechanism which is different from CSMA/CA were proposed. Nonzero sizes of IEEE 802.11 MAC backoff window were given to classthree service while zero window size was given to class-one emergency messages. In this way, emergency message gets the channel before beacon even though they are ready to broadcast at the same time because beacon has to wait for a DIFS before transmission. In [14], vehicles located in the farthest narrow segment were assigned smaller size of CW to rebroadcast packets first. 


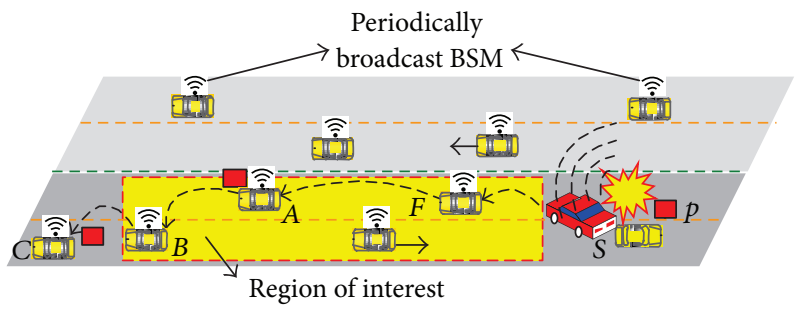

FIgURE 1: A typical highway scenario in VANET.

The common drawback of these schemes is that they view all packets the same and ignore the difference of the information quantity of messages, which is of great significance in providing vehicles with real-time and precise safety information. Some efforts $[18,19]$ have been present to differentiate packets' priority, such as EDCA scheme in 802.11p; however, they only consider only one aspect of information quantity (e.g., distance or type of messages). In addition, PVCast [20] integrates data preference into broadcast protocol. Although more data coverage and throughput can be achieved, lots of redundancy would be produced to satisfy the data preference of all vehicles in the network.

\section{Safety Information Quantity of Message: Motivation and Challenges}

In real vehicular networks, there are many kinds of safety messages to broadcast for satisfying different applications. Take a typical highway scenario as an example, as shown in Figure 1. There are two types of safety messages in the network. One is the Basic Safety Message (BSM) [21], which is broadcasted periodically to announce vehicle's status information (i.e., position, direction, velocity, neighbor number, etc.) to its neighbors for collision detecting, for instance. Another one is emergency message (EM), which is disseminated by multihop to warn the vehicles driving towards the given place where the emergency happened. We can see from the figure that an emergency message in terms of packet $p$ is forwarded hop by hop until it covers the whole RoI. With the increase of time or as the extension of space in the form of cumulative hops and distances, the emergency level of $p$ decreases. Thus its information quantity gets less and even turns to be zero when it reaches the end or out of RoI. A message with zero information quantity is useless for any vehicle. To this end, we can conclude that the information quantity of messages in vehicular network changes temporally and spatially. Besides, EM contains more information quantity than BSM in the same condition.

We then use two examples to show the necessity to consider the information quantity when designing a broadcast protocol. In Figure 2(a), two packets $p 1$ and $p 2$ are generated at the same place but at different time. Farthest-first protocols assign them the same priority to access the channel due to the same one-hop coverage. However, the newer packet $p 2$ contains more recent condition of traffic, which is more useful to the coming vehicle $F$. Therefore, to obtain more information quantity for vehicle $F$, vehicle $B$ should have a higher priority to broadcast $p 2$.

In Figure 2(b), two packets are generated at the same time but at different locations. Since $p 1$ covers longer onehop distance from current receiver to last forwarder, most existing protocols, for example, waiting-based protocols, give higher priority to vehicle $A$ to broadcast $p 1$ because $A$ can disseminate a packet farther away from its source than $B$ does. However, vehicle $F$ prefers to receive the nearby packet $p 2$ compared to $p 1$ because $p 2$ contains the status of a vehicle closer to $F$. The earlier $F$ receives $p 2$, the more information quantity $F$ can get. To obtain more accurate information of road and traffic, therefore, vehicle $B$ should have higher priority to broadcast $p 2$.

Motivated by these observations, we think it is necessary to give much attention to study the difference of information quantity of messages in VANET and to incorporate it into the design of safety data broadcast protocol. We attempt to analyze the information quantity of messages in the following three aspects.

Temporal Information Quantity. The longer duration from generation to current time a message experiences, the less information quantity it contains. New message is more important than outdated message.

Spatial Information Quantity. The larger space in terms of cumulative hop and distances a message covers from generation location to current location, the less information quantity it contains. A nearby message with fewer hops is more urgent than a farther one with more hops.

Type Information Quantity. EMs are life-critical messages while BSMs are background beacons. Hence, EM contains more information quantity than BSM.

We face the following challenges when we integrate the information quantity into the design of safety data broadcast protocol.

Challenge 1. A comprehensive model is needed to quantify the information quantity of messages. The information quantity depends on several attributes of messages, such as temporal attribute, spatial attribute, and type attribute. All these attributes should be considered completely. Ignoring any one of them will lead to severe decrease of broadcast performance, such as high delivery delay [20]. 


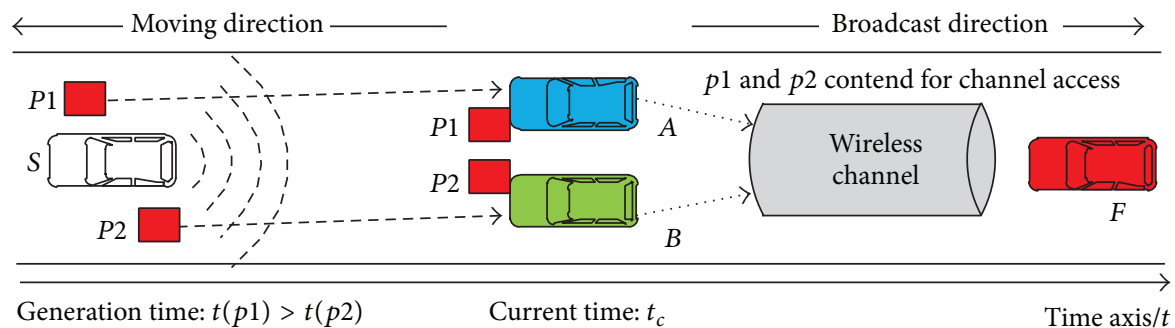

(a) Temporal information quantity

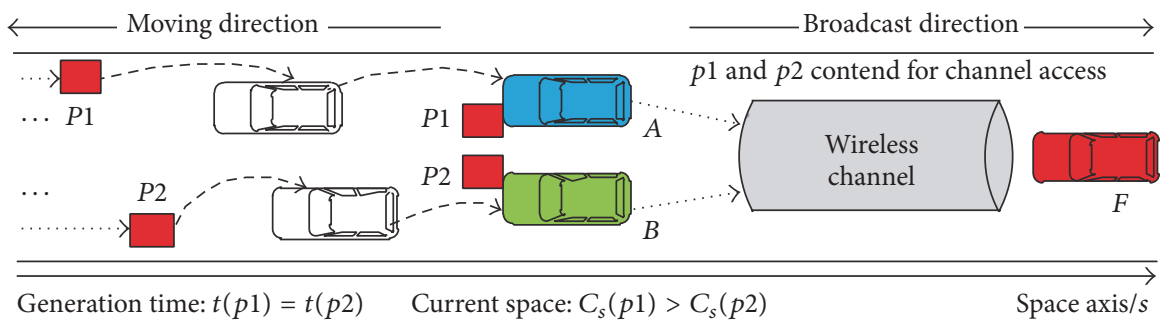

Total space: $T_{s}(p 1)>T_{s}(p 2)$

(b) Spatial information quantity

FIGURE 2: Two aspects of information quantity.

Challenge 2. Other than ensuring vehicles to receive more recent and more accurate emergent messages in time, the newly proposed protocol should address the common problems of broadcast protocols such as reliability, timeliness, redundancy, overhead, and scalability. Only in this way are the increasing kinds of safety applications likely to be supported better.

\section{Metric of Information Quantity}

In this section, we will model the information quantity to address Challenge 1 discussed in Section 3. Since the messages are disseminated in the unit of packet, the following discussion about emergency-degree modeling will be perpacket level.

Definition 1. Given a packet $p$ arriving at vehicle $v$ to be disseminated, its emergency-degree $\operatorname{ED}_{v}(p)$ indicates the value of information quantity, which depends on temporal, spatial, and type information quantity.

We explore the modeling and computation of emergencydegree in the following. As is shown in Figure 1, given an emergency packet $p$, it is generated by vehicle $S$ with the location $\left(x_{0}, y_{0}\right)$ at time $t_{0}$. After broadcasting by vehicle $S$, packet $p$ is received by vehicle $F$ at current time $t_{c}$ with current location $\left(x_{c}, y_{c}\right)$, where the message is waiting to be handled based on a forwarding rule. At this moment, the cumulative hop the packet $p$ experiences turns to be $h_{c}$ from zero. So long as the packet is not out of RoI, it should be forwarded sequentially. Then each packet can be defined by an 8-tuple $\left\{t_{0}, t_{c}, x_{0}, y_{0}, x_{c}, y_{c}, h_{c}\right.$, type $\left.{ }_{p}\right\}$. For example, once a vehicle receives a packet $p$, we can say that, during the time $\Delta t=t_{c}-t_{0}$, the type ${ }_{p}$ packet covers a distance $\operatorname{dis}_{c}=\sqrt{\left(x_{c}-x_{0}\right)^{2}+\left(y_{c}-y_{0}\right)^{2}}$ after broadcasting $h_{c}$ hop. In the procedure of multihop broadcast, the content of packets' 8-tuple changes, representing the dynamic of information quantity. For a given packet, its emergency-degree value is changing both temporally and spatially, by which the information quantity of packets is quantified. Therefore, the computation of emergency-degree should include all three classes of packets properties. Then the emergency-degree of packet $p$ at location $\left(x_{c}, y_{c}\right)$ and time instance $t_{c}$ for vehicle $v$ can be expressed as

$$
\mathrm{ED}_{v}(p)=T_{v}(p) \cdot S_{v}(p) \cdot \mathrm{TP}_{p}
$$

In formula (1), $T_{v}(p)$ is the temporal emergency-degree function of duration that packet $p$ experiences. $S_{v}(p)$ is the spatial emergency-degree function of distances and hop that packet $p$ covers. And $\mathrm{TP}_{p}$ is the type emergency-degree function of the type that packet $p$ belongs to. The product of function $T_{v}(p), S_{v}(p)$, and $\mathrm{TP}_{p}$ integrates all three packet information quantities into the signal expression of packet emergency-degree. In the following we will define these functions, respectively.

4.1. Temporal Emergency-Degree Function. The newer message can provide vehicles with recent road and traffic condition; thus it should has a higher emergency-degree value. With the increase of duration a message experiences, its emergency-degree decreases. The descent speed is high at first and gets slow gradually. Moreover, no matter how long a message experiences, it is still useful theoretically for some applications such as density estimation of vehicles. Therefore, temporal emergency-degree value is always greater than zero. In addition, since the latency of multihop EM (on the level of millisecond $[22,23])$ is much less than the update interval of 
BSM (more than $100 \mathrm{~ms}$ ), EM is always more urgent. Thus the temporal emergency-degree of EM is greater than that of BSM when they are created at the same time. From these observations, we summarize the properties of a temporal emergency-degree function which should include that

(1) $T_{v}(p)>0, d T_{v}(p) / d t_{c}<0, d^{2} T_{v}(p) / d^{2} t_{c}>0$;

(2) $T_{v}(\mathrm{EM})$ decreases slower than $T_{v}(\mathrm{BSM})$.

Formula (2) is proposed to quantify the temporal emergencydegree of a packet:

$$
T_{v}(p)=e^{-\omega_{t p}\left(t_{c}-t_{0}\right)}
$$

where $\omega_{t p}$ is the fading factor of temporal emergency-degree depending on the type of message. We define $\omega_{b}>\omega_{e}>0$, which represents that the temporal emergency-degree of EM decreases slower than that of BSM. Formula (2) differentiates different types of packets generated at different time, for example, Figure 2(a); then the emergency packets newly generated will have a higher priority.

4.2. Spatial Emergency-Degree Function. Due to channel fading and collision, many emergency packets actually experience different hop when they reach adjacent vehicles for competing channel access, although uniform forwarding rule is followed. With the increase of hops, packets become useless for the potential receivers, because the intermediate forwarders gradually expose the packets' information every hop. As a result, packets with different hop do not stay the same level of emergency, although they are generated at the same time and are covering the same distances. Vehicles prefer to receive packets with fewer cumulative hops, because these packets are of more indeterminacy. That is why spatial information quantity depends on both the message's covering distances and cumulative hop.

With the extension of space, the emergency-degree of distance and hop decreases. On one hand, the distance emergency-degree decreases as the distance increases until the message reaches out of RoI, where it becomes zero. We summarize the properties of a distance emergency-degree function which should include that

(1) DIS $(p)=0$, if vehicle $v$ moves away from generation location of packet $p$;

(2) $\operatorname{DIS}(p)=0$, if $\operatorname{dis}_{c}>d_{\mathrm{RoI}}$, where $d_{\mathrm{RoI}}$ is the range of RoI;

(3) $\operatorname{DIS}(p) \geq 0, d \mathrm{DIS}(p) / d \operatorname{dis}_{c}<0$, if $\operatorname{dis}_{c} \leq d_{\mathrm{RoI}}$.

Formula (3) is proposed to quantify the distance emergencydegree of a packet:

$$
\begin{aligned}
\operatorname{DIS}(p) & =\max \left(0,1-\frac{\operatorname{dis}_{c}}{d_{\mathrm{RoI}}}\right) \\
& =\max \left(0,1-\frac{\sqrt{\left(x_{c}-x_{0}\right)^{2}+\left(y_{c}-y_{0}\right)^{2}}}{d_{\mathrm{RoI}}}\right) .
\end{aligned}
$$

On the other hand, the hop emergency-degree degrades rapidly as the cumulative hop rises. Although hop attribute plays a small part on spatial emergency-degree, it should not be ignored, especially in the bad communication condition such as channel fading, obstructions, interference, contention, and collision. For simplicity, we assume that cumulative hop and elapsed time characterize the same reduction trend, and the property of hop emergency-degree function is similar to that of temporal emergency-degree function. From these observations above, formula (4) is proposed to quantify the hop emergency-degree of a packet:

$$
\operatorname{HOP}(p)=e^{-w_{e} h_{c}}
$$

where $w_{e}$ is the fading factor of EM.

Since hop attribute is mainly affected by communication environment, while distance attribute mainly depends on forwarding rule, no necessary relation exits between them, although they seem to follow a similar varying pattern. From these summaries above, a weight formula is proposed to evaluate a packet's spatial emergency-degree approximately:

$$
S_{v}(p)=A \cdot \operatorname{DIS}(p)+B \cdot \operatorname{HOP}(p),
$$

where $A$ and $B(A \geq 0, B \geq 0$, and $A+B=1)$ are weight factors of distance emergency-degree and hop emergencydegree, respectively. The values of $\mathrm{A}$ and $\mathrm{B}$ depend on the importance of distance and hop attributes on the spatial emergency-degree in typical scenario of VANETs.

We can see from formulas (3)-(5) that a message that covers longer range and experiences more hops becomes less urgent, containing less spatial information quantity. However, the farthest-first schemes assign higher priority to packets that only cover longer distance of current hop, overtaking the whole covered distances from generation to current location, for example, Figure 2(b). On the contrary, we assign lower priority to those packets that undergo broader space. Hence, vehicles will collect more precise safety information.

4.3. Type Information Quantity Function. As we know in EDCA, safety services have higher priority to access the channel in terms of smaller size of CW than nonsafety services. However, the type of safety messages should be further subdivided when considering their information quantity. That is because a number of safety messages $[13,24]$ would be broadcasted in VANET, acting different levels of emergency to implement all kinds of applications. Apart from affecting the reduction speed of temporal emergency-degree in (2), the type attribute also affects the initial value of packet newly generated. Thus we use different weight to quantify different types of messages:

$$
\mathrm{TP}_{p}=W_{\mathrm{TP}}, \quad \mathrm{TP} \in\{\mathrm{EM}, \mathrm{BSM}\}
$$

in which we define $W_{\mathrm{EM}}>W_{\mathrm{BSM}}>0$ to indicate that the information quantity of EM is larger than BSM. 
To the end, the emergency-degree-based information quantity can be rewritten in

$$
\begin{aligned}
& \operatorname{ED}_{v}(p) \\
& \quad= \begin{cases}{\left[A \cdot \max \left(0,1-\frac{\sqrt{\left(x_{c}-x_{0}\right)^{2}+\left(y_{c}-y_{0}\right)^{2}}}{d_{\mathrm{RoI}}}\right)+B \cdot e^{-w_{e} h_{c}}\right] \cdot e^{-\omega_{t p}\left(t_{c}-t_{0}\right)} \cdot W_{\mathrm{TP}},} & \vec{v} \in\left\{\operatorname{RoI}, \operatorname{dis}_{c}(p)<d_{\mathrm{RoI}}\right. \\
0, & \text { otherwise, }\end{cases}
\end{aligned}
$$

where $\vec{v} \in\{\mathrm{RoI}\}$ means vehicle $v$ moves towards the packet generation location with the opposite direction of broadcast. Other vehicles such as vehicles moving away from the packet generation location or vehicles on the other moving direction will be valued as zero by this model.

Emergency-Degree-Based Information Quantity. Given any two packets at vehicle $v, p 1$ will have larger information quantity than $p 2$ if $\mathrm{ED}_{v}(p 1)>\mathrm{ED}_{v}(p 2)$. In another way, given two packets at vehicle $A$ and vehicle $B$, respectively, vehicle $A$ with $p 1$ will have higher information quantity than vehicle $B$ with $p 2$ if $\mathrm{ED}_{A}(p 1)>\mathrm{ED}_{B}(p 2)$.

With these equations, we have modeled the metric of information quantity with emergency-degree, considering all three attributes. Thus, Challenge 1 discussed in Section 3 can be resolved.

\section{EDCast: An Adaptive Emergency-Degree- Based Broadcast Protocol}

Having quantified the information quantity of message with emergency-degree metric, we wander the benefits of incorporating it into the design of broadcast protocol. EDCast, an adaptive emergency-degree-based broadcast protocol, is proposed in this section.

5.1. Overview of EDCast. Farthest-first scheme is the most common protocol in VANET, in which farther vehicles are assigned higher priority in terms of less waiting time to access the channel. The waiting time is inversely proportional to the distance from receiver to the previous forwarder, which is shown as follows:

$$
\mathrm{WT}=\operatorname{MaxWT} \cdot\left(1-\frac{d}{R}\right),
$$

where $d$ is the one-hop distance from itself to the last forwarder, $R$ is the communication range, and MaxWT is the maximum waiting time.

To address Challenge 2 discussed in Section 3, we introduce the farthest-first scheme as the basic principle of EDCast for relay selection and redundancy suppression. Then we integrate information quantity into it to differentiate messages' priority. Figure 3 shows the flow chart of EDCast.
We can see from Figure 3 that once upon a new packet $p$ reaches vehicle $A$, EDCast will decide whether or not to broadcast the packet and how to disseminate it by calculating the emergency-degree $\operatorname{ED}_{v}(p)$. After updating network parameters based on the estimated number of vehicles, EDCast assigns packet $p$ appropriate waiting time WT, the size of the minimal CW, and broadcast probability $P_{r}$ according to its type. The self-adaptation scheme of network status will be described in Section 5.3.

Based on the farthest-first protocols, EDCast is lightweight without increasing any control overhead and fully distributed without the help of RSU. Thus it is feasible and convenient for EDCast to be embedded into the current structure of 802.11p.

5.2. Probability and CW Size Assignment Module. To provide vehicles with the recent and precise information, EDCast configures higher priority to the messages with higher ED values in terms of smaller size of CW. Furthermore, in order to reduce the collision among packets during transmission, EDCast randomly drops the packets with small ED value in certain probability. The probability scheme is also used to alleviate the broadcast storm especially in the dense area. Particularly, if the vehicle is the source of packet, the packet will be disseminated immediately with the initialized network parameters.

We use the following piecewise functions to evaluate the probability and CW size of a packet based on its ED value, which are shown in (9) and (10), respectively.

$$
P_{e}(p)= \begin{cases}P_{1}, & \mathrm{ED}(p) \geq \overline{\mathrm{ED}_{1}} \\ P_{2}, & \overline{\mathrm{ED}_{2}} \leq \mathrm{ED}(p)<\overline{\mathrm{ED}_{1}} \\ P_{3}, & \text { otherwise }\end{cases}
$$

where $P_{i}, i=1,2,3$, is the threshold of forwarding probability, and we predefine $1>P_{1}>P_{2}>P_{3}>0$. What is more, we also predefine the threshold of $\mathrm{ED}$ value $\overline{\mathrm{ED}_{1}}>\overline{\mathrm{ED}_{2}}>0$, based on which we differentiate the level a packet belongs to.

$$
\mathrm{CW}_{\text {min }}(p)= \begin{cases}\mathrm{CW}_{1}, & \mathrm{ED}(p) \geq \overline{\mathrm{ED}_{1}} \\ \mathrm{CW}_{2}, & \overline{\mathrm{ED}_{2}} \leq \mathrm{ED}(p)<\overline{\mathrm{ED}_{1}} \\ \mathrm{CW}_{3}, & \text { otherwise, }\end{cases}
$$

where $\mathrm{CW}_{i}, i=1,2,3$, is the threshold of size of CW. 


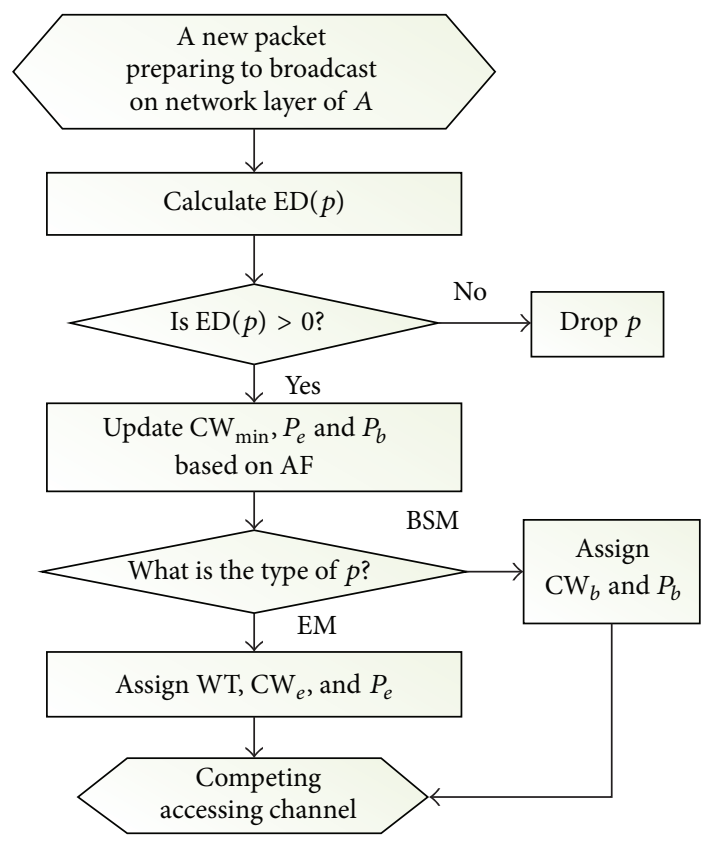

Figure 3: The flow chart of EDCast.

The piecewise functions such as (9) and (10) are necessary, for they provide protocol designers with flexibility to adjust the threshold parameters dynamically according to the network condition. In this way, an adaptation scheme could be used to optimize the broadcast performance of EDCast.

5.3. Network Adaptation Module. As known, VANET characterizes highly dynamic topology. Neither sparse network nor dense network will decrease broadcast performances significantly if the networks parameters remain constant. An adaptation scheme, just as a simple example, is proposed to adjust these parameters roughly. In EDCast, the exponentially weighted moving average (EWMA) $[20,25]$ traffic density estimator is used to estimate $\widehat{N}_{A}$, the real-time vehicle number within the one-hop coverage of vehicle $A$. During the period $T$ (e.g., $5 \mathrm{~s}$ ), EDCast counts the number of vehicles $A$ can hear. Then $\widehat{N}_{A}$ can be estimated as the following equation:

$$
\widehat{N}_{A}[T]=\alpha \cdot \widehat{N}_{A}[T-1]+(1-\alpha) \cdot N_{A}[T],
$$

where $\alpha$ is the weight factor and $N_{A}[T]$ is the average number of neighbors $A$ is able to hear during $T$.

After getting the estimated number of vehicles, we define the adaptation factor (AF) for each vehicle to adjust broadcasting parameters dynamically when they have packets to disseminate.

Definition 2. Given a vehicle $A$, the adaptation factor (AF) is the estimated number of vehicles $A$ divided by the maximum number $A$ can hear in theoretical.
The AF value can roughly reflect the changing density around each vehicle, which is also per-packet level:

$$
\mathrm{AF}_{v}=w_{\mathrm{sc}} \cdot \frac{\widehat{N}_{v}}{\widehat{N}_{\mathrm{MAX}}},
$$

where $\widehat{N}_{\text {MAX }}$ is the maximum number of neighbors within the communication range of vehicle $v$, which is calculated by $\widehat{N}_{\mathrm{MAX}}=\left(2 * R /\left(L+l_{\text {safe }}\right)\right) * N_{\text {lane }}$, where $R$ is intended communication range; $L$ is the average length of vehicle; and $l_{\text {safe }}$ is the safety length, the average distance between vehicles when they are locating one by one safely in the most heavy traffic. And $N_{\text {lane }}$ is the number of lanes. $\widehat{N}_{v}$ is the estimated number of neighbors of vehicle $v$. And $W_{\mathrm{sc}}$ is the weight factor depending on the chosen simulation scenario, which is used to balance the value of $\operatorname{AF} \in(0,1)$.

For simplicity, a piecewise function is introduced to adjust the network parameters reasonably according to the estimated adaptation factor of each vehicle. The range of AF is divided into three parts, each of which represents the average level of traffic condition in certain scenario. In our simulation scenario, $\mathrm{CW}_{\min }$ and MaxWT are directly proportional to adaption factor $\mathrm{AF}$, while $P_{i}$ is inversely proportional to AF. Based on the estimated value of AF, three groups of parameters among parameters pool of $\mathrm{CW}_{G i}$ and $P_{G i}$ are configured to $\mathrm{CW}_{\min }$ and $P_{i}$, respectively, while three single levels of parameters are assigned to MaxWT as well, which are shown in

$$
\begin{aligned}
& \left(\mathrm{CW}_{\min }^{G}, P_{i}^{G}\right) \\
& = \begin{cases}\left(\left\{\mathrm{CW}_{G 1}\right\},\left\{P_{G 1}\right\}\right), & 0 \leq \mathrm{AF} \leq \mathrm{AF}_{1} \\
\left(\left\{\mathrm{CW}_{G 2}\right\},\left\{P_{\mathrm{G} 2}\right\}\right), & \mathrm{AF}_{1} \leq \mathrm{AF} \leq \mathrm{AF}_{2} \\
\left(\left\{\mathrm{CW}_{G 3}\right\},\left\{P_{\mathrm{G}}\right\}\right), & \mathrm{AF}_{2} \leq \mathrm{AF} \leq 1,\end{cases} \\
& \operatorname{MaxWT}= \begin{cases}\mathrm{WT}_{1}, & 0 \leq \mathrm{AF} \leq \mathrm{AF}_{1} \\
\mathrm{WT}_{2}, & \mathrm{AF}_{1} \leq \mathrm{AF} \leq \mathrm{AF}_{2} \\
\mathrm{WT}_{3}, & \mathrm{AF}_{2} \leq \mathrm{AF} \leq 1 .\end{cases}
\end{aligned}
$$

The detailed configure will be given in the section of simulation. In this way, the greater section AF belongs to, the larger $\mathrm{CW}_{i}$ and MaxWT are and the smaller $P_{i}$ is.

As is known, the background safety message BSM plays a vital part in providing vehicles' status in realistic VANET, on which many safety applications rely, such as crash detection and density estimation. The more frequent the BSM sends, the more recent and precise status information of vehicles it presents. However, too frequent BSMs will cause server contention for channel access, leading to unreliable transmission. Therefore, there is a trade-off between broadcast performance and the accuracy of vehicle number estimation, which deserves much attention from protocol designers. As a matter of fact, with the increase of vehicle density, vehicles move more slowly, and the relative velocity between vehicles gets smaller, even approaching zero. The status of vehicles in these conditions remains the same for a long duration; thus 


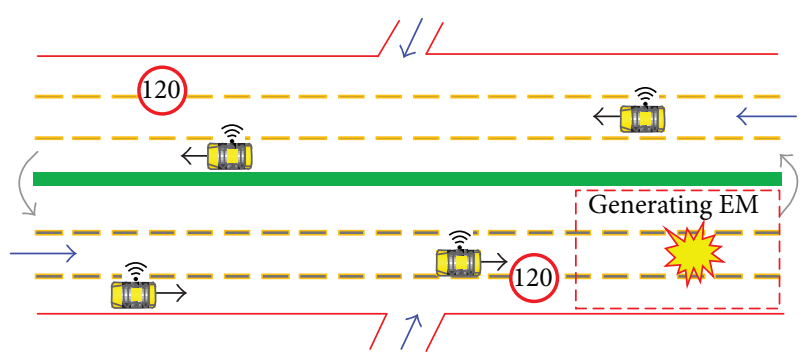

FIGURE 4: A typical highway scenario for simulation.

it is necessary to adjust the frequency of BSMs dynamically. In addition, the proposed adaption scheme in this paper is only a simple example to inspire better ideas for improving EDCast in the future.

\section{Performance Evaluation}

The network simulator NS2.35 is used to evaluate the performance of EDCast. EDCast is embedded as a middle-layer protocol between IEEE-802.11p MAC protocol and upper application protocol. When deciding to disseminate a packet, the configured minimum size of $\mathrm{CW}$ of this packet is passed from EDCast to MAC layer in the packet header. In this way, we do not need any intrusive change to the current IEEE802.11p protocol stack. Besides, it is portable for EDCast to be installed into different protocol stacks.

\subsection{Simulation Environment}

Simulation Scenario. A traffic simulator, Simulation of Urban Mobility (SUMO) [26], is used to generate the trace of vehicles for a typical highway scenario shown in Figure 4. The topology consists of a bidirectional road of $2000 \mathrm{~m}$ long and $40 \mathrm{~m}$ wide with 6 lanes. There are two entrances in each moving direction: one is at the start and the other one is at the middle. 20-100 vehicles, including four types with different acceleration, deceleration, length, and limited speed, enter the topology successively at a period of 1 second from the four entrances, respectively. All the vehicles move following the modified Krauss Model. A connection file is configured to guide the vehicles driving encircling the bidirectional road, entering the reverse road when exiting from one road. Besides, $5 \%$ of the vehicles locate on the road uniformly and move slowly for composing a connected topology.

Communication Configuration. The communication range of each vehicle is set to be $300 \mathrm{~m}$, and the range of RoI of emergency messages is set to be $1000 \mathrm{~m}$. Multihop broadcast is necessary for EMs to cover the whole RoI. Vehicles locating at certain range (e.g., range between 1500 and $2000 \mathrm{~m}$ ) generate EMs every 1 second with a certain probability (e.g., 50\%). Vehicles in the network are both message generators and message forwarders. 10 BSMs are initialized to be generated every 1 second by each vehicle to announce its status. The main parameters for simulation are shown in Table 1.
TABLE 1: Simulation parameters of scenario.

\begin{tabular}{lc}
\hline Parameter & Value \\
\hline Topology scenario & $2000 \mathrm{~m} \times 40 \mathrm{~m}$ \\
Lanes, bidirectional & 6 \\
Number of vehicles & $20,40,60,80,100$ \\
Speed range & $30 \sim 120 \mathrm{~km} / \mathrm{h}$ \\
Communication range & $300 \mathrm{~m}$ \\
$d_{\text {RoI }}$ & $1000 \mathrm{~m}$ \\
EM size & 300 bytes \\
PHY model & TwoRayGround \\
MAC model & $802.11 \mathrm{p}$ \\
Simulation time & 200 seconds \\
\hline
\end{tabular}

To illustrate the necessity of considering the information quantity of messages to improve broadcast efficiency, we comparatively study the following protocols in the same scenario.

Mflood. The most original receiver-based protocol is implemented into VANET. Once they receive a packet, vehicles in mflood forward it immediately if the packet is new. Broadcast storm in the scenario of dense network needs to be optimized.

Farthest. The farthest-first protocol is first proposed in [6] for VANET. The vehicles that are farther to sender are assigned higher priority to access the channel in terms of less waiting time. In this way, during the procedure of multihop broadcast, one-hop forwarding can achieve higher geographical progress and less latency. That is why farthest is suggested as the basic idea of many protocols.

Slotted-p. A typical probability-based protocol. Similar to farthest, slotted-p designs a waiting timer to schedule packets based on the distance between the sender and receiver. When the waiting timer expires, the scheduler disseminates the packet with a probability (e.g., 50\%) if no duplicates are received. Redundancy is reduced significantly.

EDCast. An adaptive emergency-degree-based protocol is presented in Section 5, which assigns more urgent messages higher priority by configuring higher probability and smaller size of CW, for the purpose of providing vehicles with the recent and precise safety information. The main communication parameters are listed in Table 2.

The following metrics are evaluated for comprehensively understanding the benefits of EDCast.

Delivery Delay. It is the average duration per hop per packet from generation to reception.

Redundancy. It is the total number of packets which are forwarded per vehicle divided by the total number of packets received.

Broadcast Efficiency. It is the total number of packets received by nodes divided by the total number of packets which are generated by sources during the simulation. 
TABLE 2: Communication parameters of vehicles.

\begin{tabular}{lc}
\hline Parameter & Value \\
\hline Average length of vehicle & $4 \mathrm{~m}$ \\
Safety length between vehicles & $2 \mathrm{~m}$ \\
Temporal fading factors $w_{b}, w_{e}$ & $0.5,0.8$ \\
Spatial weight factors $A, B$ & $0.4,0.6$ \\
Type weight factors $W_{\mathrm{BSM}}, W_{\mathrm{EM}}$ & 2,10 \\
MaxWT $_{i}$ & $\{5,15,25\} \mathrm{ms}$ \\
Emergency-degree threshold $\mathrm{ED}_{i}$ & $\{4,8\}$ \\
Broadcast probability $P_{\mathrm{Gi}}$ & $P_{\mathrm{BSM}}=\{0.02,0.05,0.1,0.2,1\}$ \\
& $P_{\mathrm{EM}}=\{0.1,0.3,0.5,0.7,1\}$ \\
Minimum size of CW & $\{31,23,15,7,3\}$ \\
Adaptation factor threshold $\mathrm{AF}_{i}$ & $\{0.3,0.7\}$ \\
Weight factor $w_{\mathrm{sc}}$ & 6 \\
\hline
\end{tabular}

Packet Delivery Ratio of Emergency (e-PDR). It is the percentage of packets covering the whole RoI successfully among the total packets generated by sources.

Broadcast Efficiency of Emergency. It is the total number of emergency packets received by nodes divided by the total number of packets which are generated by sources during the simulation.

Delivery Delay of Emergency. It is the average delivery delay of the first three hops of emergency message.

The first three metrics are regularly known in other literatures while the other three metrics are special for emergency messages in this paper.

6.2. Simulation Results. We simulate every scenario 10 times with different initialized values and get the average values of each metric during the 200-second simulation time. In the following, we present the simulation results of all the six metrics.

Figure 5 shows the average one-hop delivery delay of all packets. We can see that it increases with the density of vehicles due to more intense contention among vehicles to access the shared channel. But the increasing speed of EDCast is smaller than that of three other protocols. When there are 20-40 vehicles in the network, the difference of delivery delay of all protocols is small, while in the scenario of $60-$ 100 vehicles existing, the delivery delay of EDCast is 3 times less than the second best one. The improvement of latency performance of EDCast mainly comes from two aspects: (1) the size of $\mathrm{CW}$ of packets is differentiated according to their emergency-degree value other than remaining the same; therefore, the contention and collision among packets are reduced significantly. (2) With the help of adaptation scheme, the maximal waiting time is changing along with the density of vehicles, which reduces the chance of unnecessary waiting. EDCast enables fast dissemination, providing vehicles with more recent safety information.

We then study the average redundancy and broadcast efficiency of all packets, as shown in Figures 6 and 7, respectively.

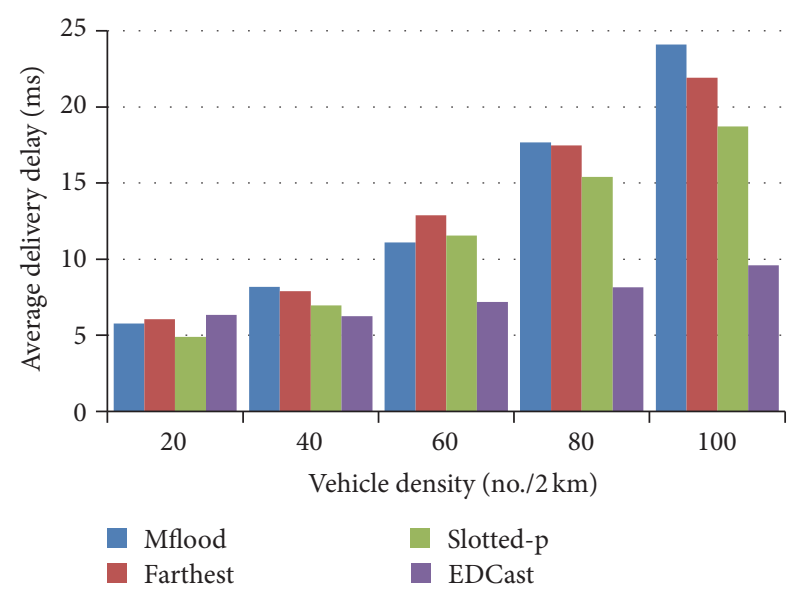

Figure 5: Delivery delay of all packets.

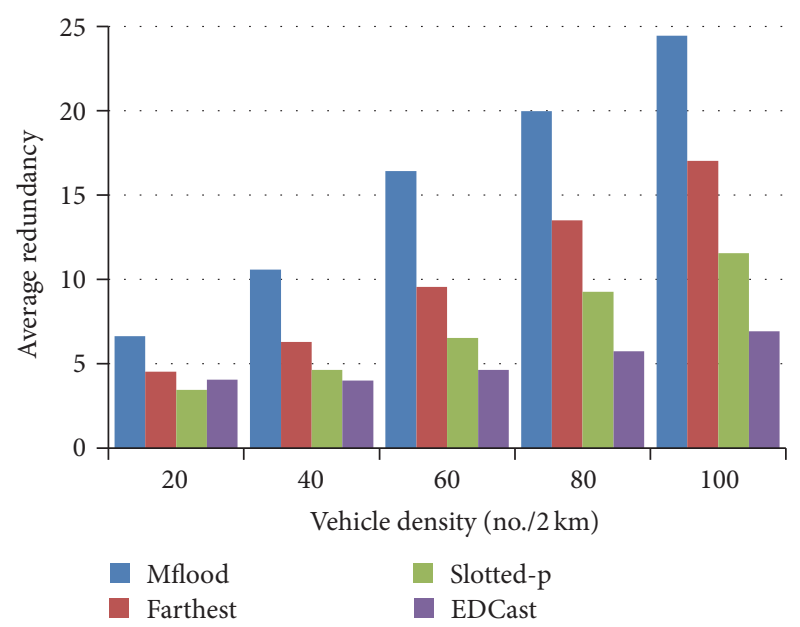

Figure 6: Redundancy.

Figure 6 shows that redundancy of all protocols increases with the number of vehicles due to more and more duplicates taking part in forwarding. In the sparse network, for example, 20 vehicles in the highway, EDCast ensures the transmission reliability by increasing dissemination probability, yielding a little more redundancy than the best one, slotted-p. But the redundancy of EDCast is still less than that of farthest and mflood. Moreover, when more than 20 vehicles exist in the highway, the redundancy of EDCast is $1 x-2 x$ less than that of three other protocols. We then plot the average broadcast efficiency of all packets in Figure 7. We can see that with the increase of vehicles the broadcast efficiency of protocols such as mflood, farthest, and slotted-p increases at first, reaches its peak when there are 40-60 vehicles, and then decreases gradually due to significant contention and collision, leading to frequent packet loss. On the contrary, by adjusting the broadcast probability and the size of the minimal CW adaptively based on the emergency-degree, EDCast disseminates packets orderly and quickly. Hence, broadcast efficiency of EDCast is much higher than that of other protocols especially in the 80-100 vehicles cases. What Figures 5-7 show illustrates that EDCast satisfies fast 


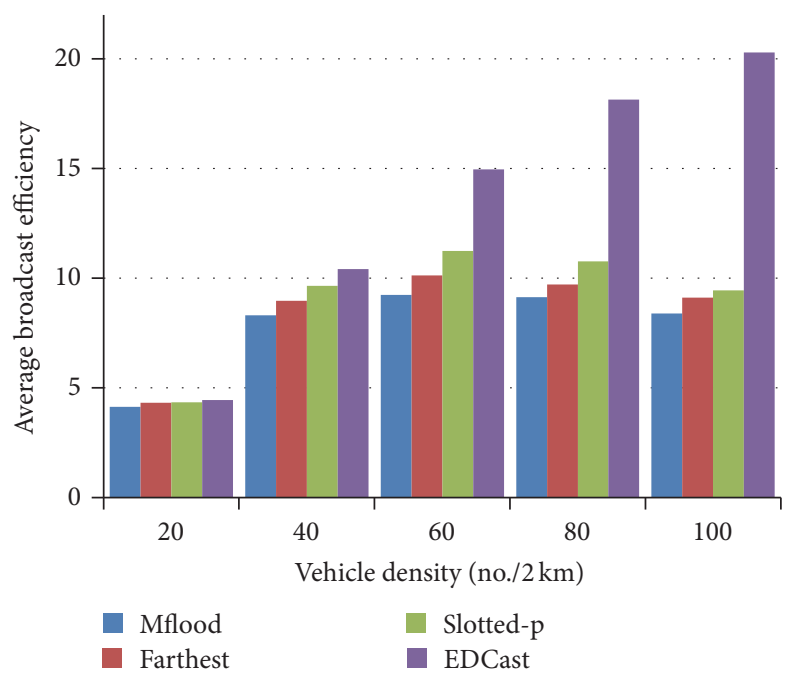

Figure 7: Broadcast efficiency.

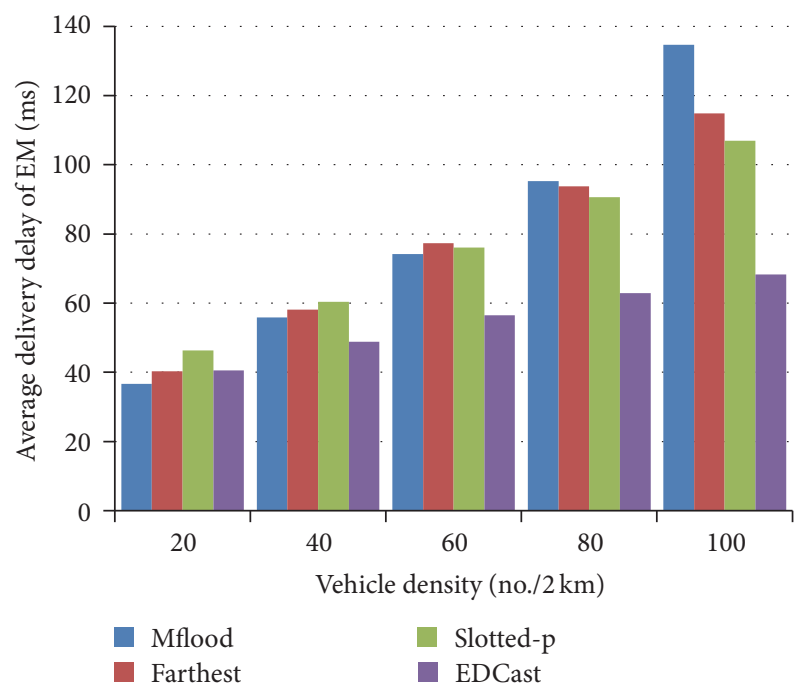

Figure 8: Delivery delay of the first three hops of emergency packets.

dissemination in various traffic conditions and alleviates broadcast storm as well.

Furthermore, we study the broadcast performance of emergency messages separately. Figures 8-10 show the average delivery delay of the first three hops, the average emergency packet delivery ratio, and the average broadcast efficiency of emergency packet, respectively. Similar to Figure 5, we can see from Figure 8 that the average delivery delay of the first three hops of EDCast is much less than that of other three protocols. We also can see from Figure 8 that the broadcast efficiency of emergency packets of EDCast is much higher than other protocols. What is more, both the improvement of the first three-hop delivery delay and emergency broadcast efficiency are larger than that of one-hop delivery delay in Figure 5 and broadcast efficiency for all packets in Figure 7.

We plot the e-PDR of all protocols in Figure 10, which represents the reliability of broadcast protocols. Unlike other

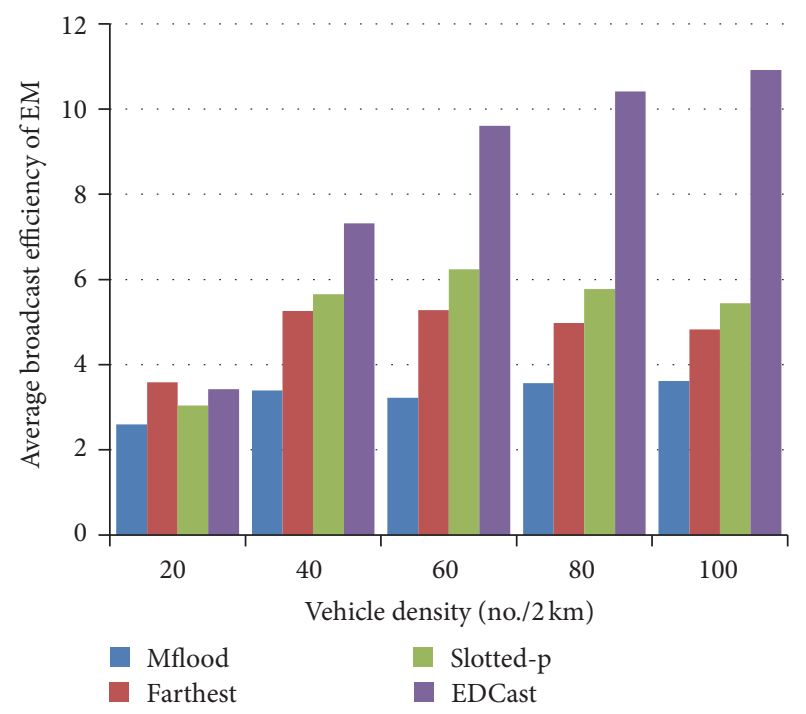

FIgURE 9: Broadcast efficiency of emergency packets.

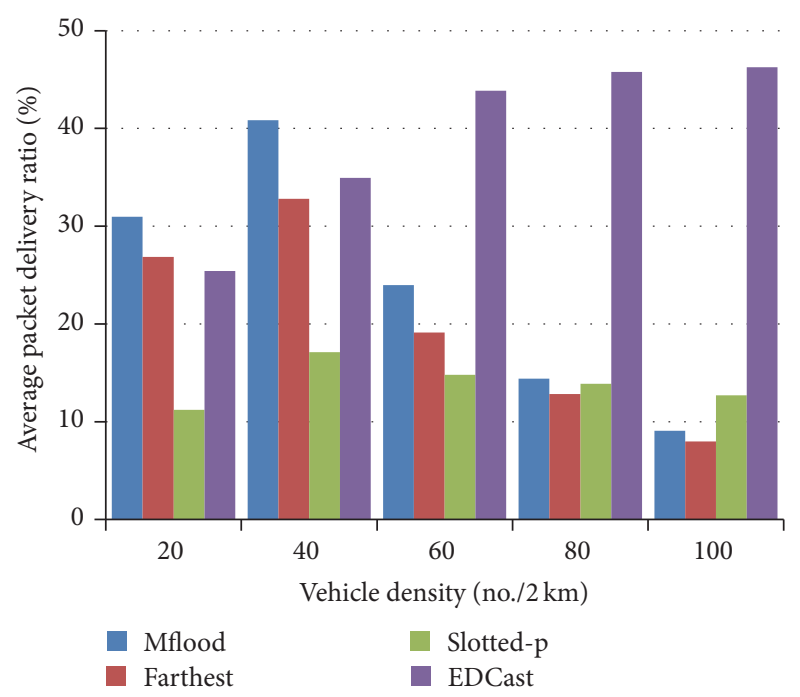

FIGURE 10: Packet delivery ratio of emergency packets.

protocols reaching the maximal e-PDR in the case of 40 vehicles and decreasing with the increase of vehicles, EDCast reaches its peak when there are 60 vehicles in the network and keeps steady afterwards. Besides, in the sparse network, for example, 20-40 vehicles, e-PDR of mflood and farthest are litter higher than EDCast, while in the scenario of dense network, for example, cases with more than 60 vehicles, EDCast performs better ( $3 x-4 x$ times) than other protocols. That is because EDCast allows only certain vehicles in the farthest segment to forward packets to suppress redundancy. So the probability for packets to cover all the vehicles in RoI is less especially in the sparse region, leading to lower ePDR of EDCast. In the crowed traffic condition of VANET, there are so many vehicles to forward packets that collision occurs frequently, leading to significant degradation of ePDR in mflood and farthest. On the contrary, by adaptively adjusting forwarding probability and size of CW, EDCast 
gets increasing e-PDR. It is demonstrated that it is reliable and efficient for EDCast to disseminate packets based on the metric of emergency-degree.

It is worth mentioning that though slotted-p produces smaller redundancy and lower deliver delay than farthest by forwarding packets in certain probability, it performs much lower e-PDR. On the contrary, EDCast reaches high broadcast efficiency and less delivery delay while introducing small redundancy at the same time. This phenomenon shows that using fixed probability and the same size of CW for broadcasting all packets is insufficient. Therefore, it is necessary to differentiate packets' probability and the minimal CW adaptively as EDCast does.

In summary, we illustrate the feasibility and effectiveness of broadcasting packets based on the information quantity for safety applications through simulation under a typical highway scenario. Compared to three other representative broadcast schemes, EDCast yields a higher broadcast efficiency and a less end-to-end delay with a less redundancy. Besides, it reaches a higher packet delivery ratio of emergency messages while consuming a lower delivery delay of the first three hops. In conclusion, EDCast can provide vehicles recent and precise safety information on road and traffic reliably.

\section{Conclusions}

In this paper, we study the influence of information quantity on broadcast performance. We propose the concept of emergency-degree to evaluate packets' emergency level on temporal, spatial, and type information quantity. EDCast, an adaptive emergency-degree-based broadcast protocol, is presented to disseminate safety messages, providing vehicles with recent and precise safety information about road and traffic. We compare the performance of EDCast with those of three other representative broadcast strategies by simulation under a typical highway scenario. The results show that EDCast obtains a substantial improvement on latency, broadcast efficiency, redundancy, and emergency packet delivery ratio. Our work proves that it is effective and feasible to design broadcast protocols based on safety information quantity of messages. In the future, we will consider the network load in the design of adaption scheme based on EDCast for improving broadcast performance.

\section{Competing Interests}

The authors declare that there is no conflict of interests regarding the publication of this paper.

\section{Acknowledgments}

The authors thank Xi Chen for constructive advice during their work. This work is supported in part by the National Great Science Specific Project of China under Grant no. 2015ZX03003012 and National Natural Science Foundation of China under Grant no. 61271184 and no. 61571065.

\section{References}

[1] W. Wang, S. S. Liao, X. Li, and J. S. Ren, "The process of information propagation along a traffic stream through intervehicle communication," IEEE Transactions on Intelligent Transportation Systems, vol. 15, no. 1, pp. 345-354, 2014.

[2] IEEE 802.11 Working Group, "Part 11: wireless LAN medium access control (MAC) and physical layer (PHY) specifications," ANSI/IEEE Std 802.11, 1999.

[3] "Standard Specification for Telecommunications and Information Exchange Roadside and Vehicle Systems-5 GHz Band Dedicated Short Range Communications (DSRC) Medium Access Control (MAC) and Physical Layer (PHY) Specifications," April 2009.

[4] J. A. Sanguesa, M. Fogue, P. Garrido, F. J. Martinez, J.-C. Cano, and C. T. Calafate, "A survey and comparative study of broadcast warning message dissemination schemes for VANETs," Mobile Information Systems, vol. 2016, Article ID 8714142, 18 pages, 2016.

[5] S. Panichpapiboon and W. Pattara-Atikom, "A review of information dissemination protocols for vehicular ad hoc networks," IEEE Communications Surveys and Tutorials, vol. 14, no. 3, pp. 784-798, 2012.

[6] L. Briesemeister and G. Hommel, "Role-based multicast in highly mobile but sparsely connected ad hoc networks," in Proceedings of the 1st Annual Workshop on Mobile and Ad Hoc Networking and Computing (MobiHOC '00), pp. 45-50, IEEE, Boston, Mass, USA, 2000.

[7] N. Wisitpongphan, O. K. Tonguz, J. S. Parikh, P. Mudalige, F. Bai, and V. Sadekar, "Broadcast storm mitigation techniques in vehicular ad hoc networks," IEEE Wireless Communications, vol. 14, no. 6, pp. 84-94, 2007.

[8] J. Sahoo, E. H.-K. Wu, P. K. Sahu, and M. Gerla, "Binarypartition-assisted MAC-layer broadcast for emergency message dissemination in VANETs," IEEE Transactions on Intelligent Transportation Systems, vol. 12, no. 3, pp. 757-770, 2011.

[9] F. J. Martinez, M. Fogue, M. Coll, J.-C. Cano, C. Calafate, and P. Manzoni, "Evaluating the impact of a novel warning message dissemination scheme for VANETs using real city maps," in NETWORKING 2010: 9th International IFIP TC 6 Networking Conference, Chennai, India, May 11-15, 2010. Proceedings, vol. 6091 of Lecture Notes in Computer Science, pp. 265-276, Springer, Berlin, Germany, 2010.

[10] H. Yoo and D. Kim, "ROFF: RObust and fast forwarding in vehicular ad-hoc networks," IEEE Transactions on Mobile Computing, vol. 14, no. 7, pp. 1490-1502, 2015.

[11] A. Mohammed, M. Ould-Khaoua, L. M. Mackenzie, and J.-D. Abdulai, "Dynamic probabilistic counter-based broadcasting in mobile Ad Hoc networks," in Proceedings of the 2nd International Conference on Adaptive Science and Technology (ICAST '09), pp. 120-127, Accra, Ghana, December 2009.

[12] A. M. Vegni, A. Mostafa, and D. P. Agrawal, "CAREFOR: collision-aware reliable forwarding technique for vehicular ad hoc networks," in Proceedings of the International Conference on Computing, Networking and Communications (ICNC '13), pp. 773-777, IEEE, San Diego, Calif, USA, January 2013.

[13] X. Ma, J. Zhang, X. Yin, and K. S. Trivedi, "Design and analysis of a robust broadcast scheme for VANET safety-related services," IEEE Transactions on Vehicular Technology, vol. 61, no. 1, pp. 46-61, 2012.

[14] K. A. Hafeez, L. Zhao, Z. Liao, and B. N.-W. Ma, "A new broadcast protocol for vehicular ad hoc networks safety applications," 
in Proceedings of the 53rd IEEE Global Communications Conference (GLOBECOM '10), pp. 1-5, Miami, Fla, USA, December 2010.

[15] W. Wang and T. Luo, “The minimum delay relay optimization based on nakagami distribution for safety message broadcasting in Urban VANET," in Proceedings of the IEEE Wireless Communications and Networking Conference (WCNC '16), IEEE Press, Duha, Qatar, 2016.

[16] M. Barradi, A. S. Hafid, and S. Aljahdali, "Highway multihop broadcast protocols for vehicular networks," in Proceedings of the IEEE International Conference on Communications (ICC '12), pp. 5296-5300, IEEE Press, Ottawa, Canada, June 2012.

[17] M. Li, K. Zeng, and W. Lou, "Opportunistic broadcast of eventdriven warning messages in Vehicular Ad Hoc Networks with lossy links," Computer Networks, vol. 55, no. 10, pp. 2443-2464, 2011.

[18] R. S. Schwartz, A. E. Ohazulike, C. Sommer, H. Scholten, F. Dressler, and P. Havinga, "Fair and adaptive data dissemination for traffic information systems," in Proceedings of the IEEE Vehicular Networking Conference (VNC '12), pp. 1-8, IEEE, Seoul, South Korea, November 2012.

[19] B. Ducourthial, Y. Khaled, and M. Shawky, "Conditional transmissions: performance study of a new communication strategy in VANET,' IEEE Transactions on Vehicular Technology, vol. 56, no. 6, pp. 3348-3357, 2007.

[20] Q. Xiang, X. Chen, L. Kong, L. Rao, and X. Liu, "Data preference matters: a new perspective of safety data dissemination in vehicular ad hoc networks," in Proceedings of the 34th IEEE Annual Conference on Computer Communications and Networks (IEEE INFOCOM '15), pp. 1149-1157, Kowloon, Hang Kong, May 2015.

[21] J. B. Kenney, "Dedicated short-range communications (DSRC) standards in the United States," Proceedings of the IEEE, vol. 99, no. 7, pp. 1162-1182, 2011.

[22] M. Torrent-Moreno, S. Corroy, F. Schmidt-Eisenlohr, and H. Hartenstein, "IEEE 802.11-based one-hop broadcast communications: understanding transmission success and failure under different radio propagation environments," in Proceedings of the 9th ACM Symposium on Modeling, Analysis and Simulation of Wireless and Mobile Systems (ACM MSWiM '06), pp. 68-77, Malaga, Spain, October 2006.

[23] X. Ma and X. Chen, "Delay and broadcast reception rates of highway safety applications in vehicular Ad Hoc networks," in Proceedings of the IEEE Workshop on Mobile Networks for Vehicular Environments (INFOCOM '07), pp. 85-90, Anchorage, Alaska, May 2007.

[24] Society of Automotive Engineers DSRC Committee, SAE J2735 Dedicated Short Range Communications (DSRC) Message Set Dictionary, 2016.

[25] I.-C. Chang, H.-T. Tai, F.-H. Yeh, D.-L. Hsieh, and S.-H. Chang, "A VANET-based $\mathrm{A}^{*}$ route planning algorithm for travelling time- and energy-efficient GPS navigation app," International Journal of Distributed Sensor Networks, vol. 2013, Article ID 794521, 14 pages, 2013.

[26] "Simulation of urban mobility (sumo)," http://sumo-sim.org/. 

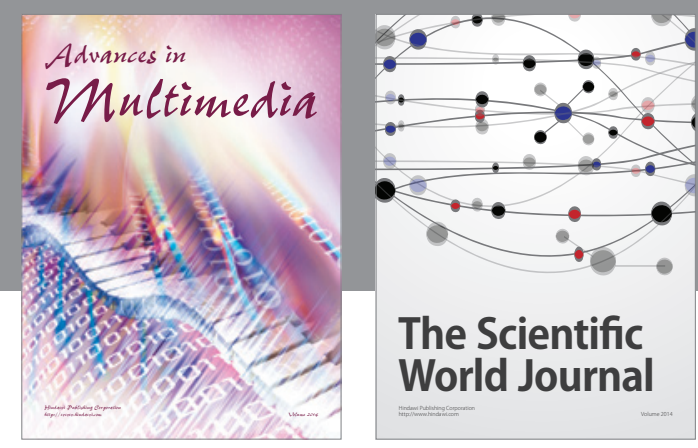

The Scientific World Journal
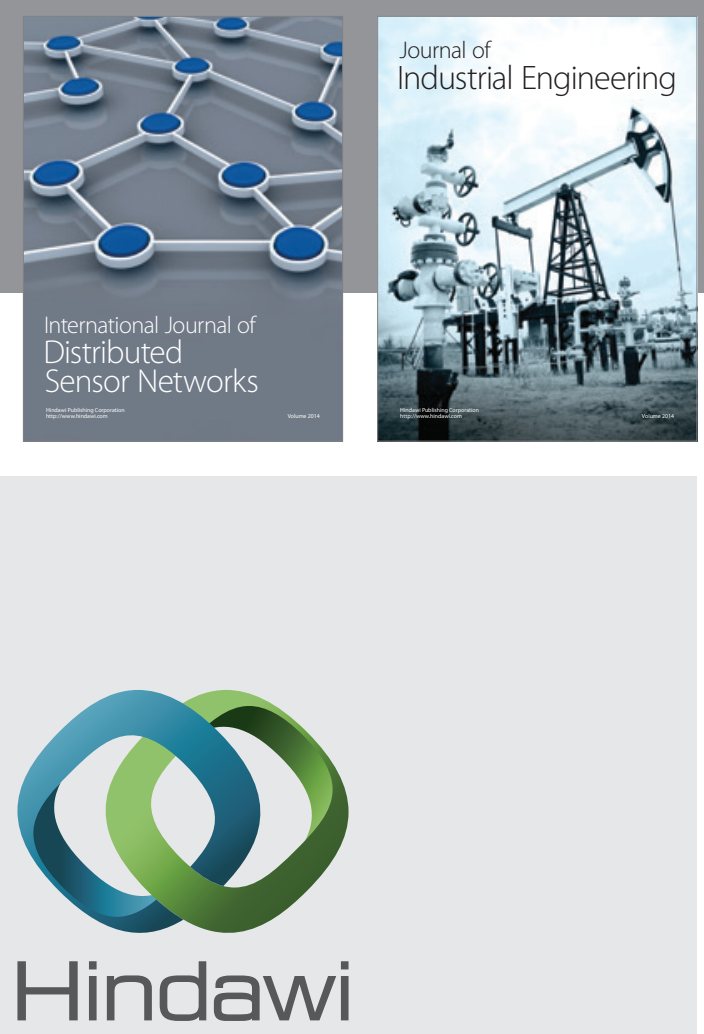

Submit your manuscripts at

http://www.hindawi.com

\section{Computer Networks} and Communications
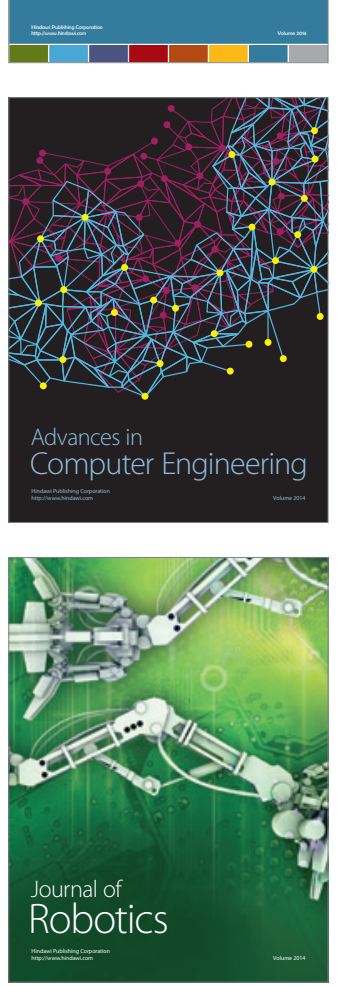
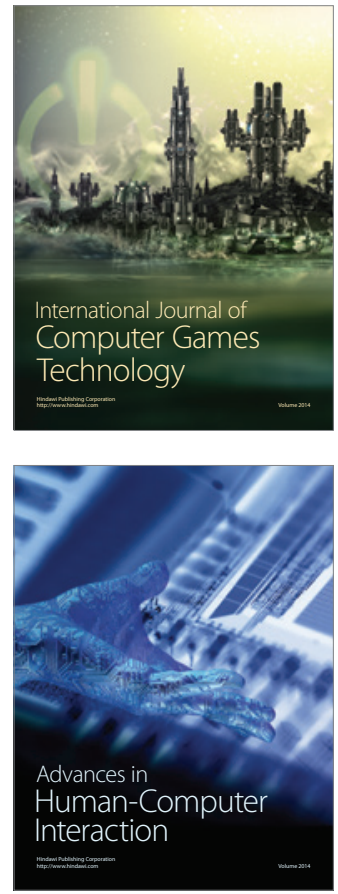
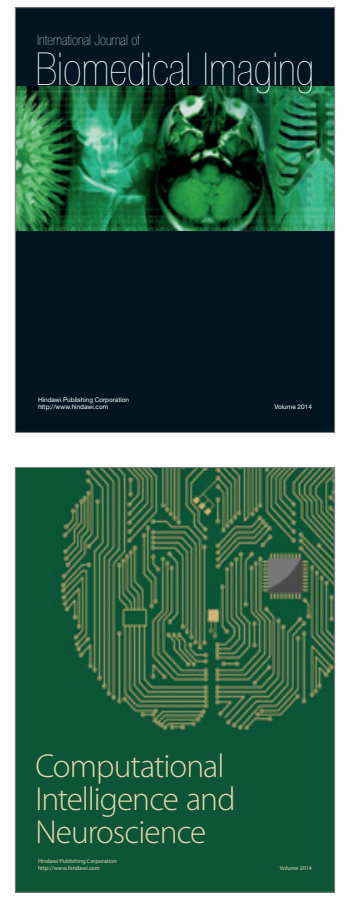
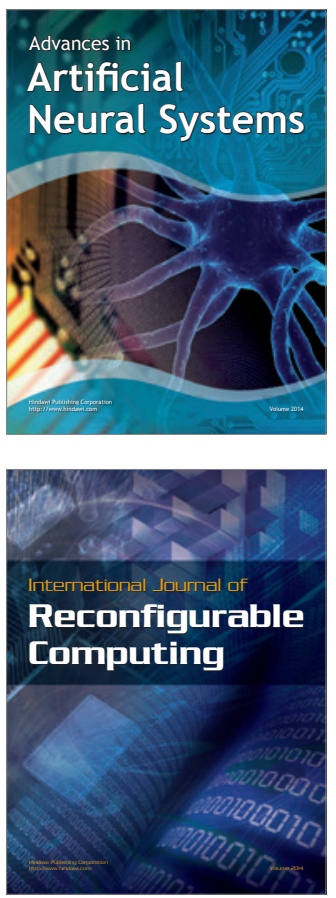
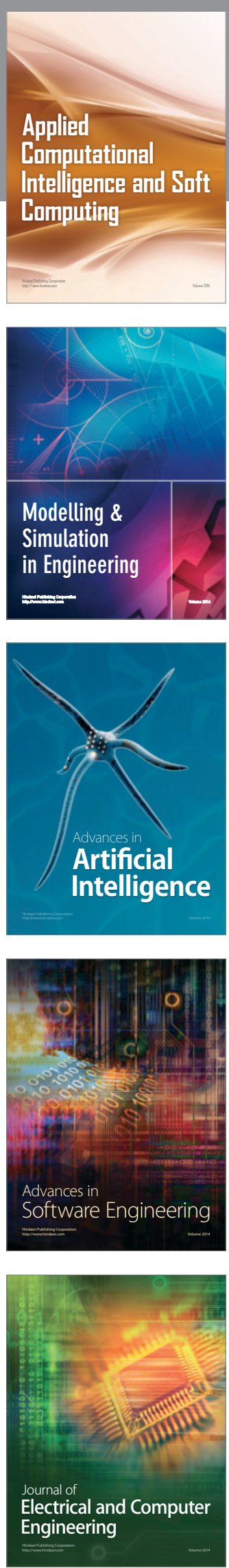